\section{Dausset, Jean}

K. Kleesiek ${ }^{1}$, C. Götting ${ }^{2}$, J. Diekmann ${ }^{3}$, J. Dreier ${ }^{4}$ und M. Schmidt ${ }^{5}$

${ }^{1}$ Ehemaliger Direktor des Instituts für Laboratoriums- und Transfusionsmedizin, Herz- und Diabeteszentrum NordrheinWestfalen Ruhr-Universität Bochum, Bad Oeynhausen,

Deutschland

${ }^{2}$ MVZ Labor Limbach Nürnberg GmbH, Nürnberg, Deutschland

${ }^{3}$ Institut für Laboratoriums- und Transfusionsmedizin, Herzund Diabeteszentrum NRW, Bad Oeynhausen, Deutschland ${ }^{4}$ Herz- und Diabeteszentrum Nordrhein-Westfalen; Institut für Laboratoriums- und Transfusionsmedizin, Universitätsklinik der Ruhr-Universität Bochum, Bad Oeynhausen, Deutschland

${ }^{5}$ Institut für Laboratoriums- und Transfusionsmedizin, Universitätsklinik der Ruhr-Universität Bochum, Bad Oeynhausen, Deutschland

Lebensdaten Französischer Arzt, geboren am 19. Oktober 1916 in Toulouse, gestorben am 06. Juni 2009 in Palma de Mallorca.

Verdienste Jean Dausset, Sohn eines Arztes, studierte an der Universität von Paris Medizin und ging im Jahr 1937 als
Assistenzarzt an ein Städtisches Krankenhaus in Paris. Im Jahr 1939 wurde er zur Armee eingezogen und diente im medizinischen Corps in Nordafrika, 1944 promovierte er in Paris im Fach Transfusionsmedizin, anschließend spezialisierte er sich auf den Bereich Hämatologie.

Im Jahr 1946 wurde er zum Doktor des Nationalen Französischen Bluttransfusionszentrums in Paris berufen, wo er bis 1963 blieb. Danach folgte er einem Ruf an die Fakultät für Medizin und wurde Professor am Lehrstuhl für Hämatologie an der Universität von Paris. Von 1969-1977 war er Professor für Immunhämatologie an der Fakultät Lariboisiere-Saint-Louis und von 1968-1984 gleichzeitig Direktor des Forschungsbereichs Immungenetik am Nationalen Medizinischen Forschungsinstitut. Von 1978-1988 ging er als Professor für Experimentalmedizin an das Collège de France.

Er erhielt im Jahr 1980 gemeinsam mit Baruh Benacerraf und George Davis Snells den Nobelpreis für Medizin und Physiologie und 1977 den Robert-Koch-Preis. Zusammen mit seinen beiden Kollegen beschäftigte er sich maßgeblich mit der immunologischen Verträglichkeit von Geweben nach Transplantationen. Sie konnten nachweisen, dass diese Immunfaktoren genetisch fixiert sind. Im Jahr 1958 definierte er das erste Lymphozytenantigen, einen Grundpfeiler des heutigen HLA-Systems ( $\triangleright$ HLA-Allele) und gilt damit als Wegbereiter der Organtransplantationen. 